\title{
ATTACHMENT FOR A TUDOR THOMAS CORNEAL GRAFT STAND*
}

\author{
BY \\ C. R. KANAGASUNDARAM \\ Eye Infirmary, Wolverhampton
}

DoNOR material for corneal grafting is not readily available. In exploring all sources, it was thought that one could use material which would otherwise be wasted if it were possible to cut satisfactory lamellar grafts from excised corneae. The attachment to be described is the result of experiments conducted with this purpose in view.

The idea originated from a desire to use corneae from eyes eviscerated after injuries. This type of material is sometimes available in industrial areas where eyes can be hopelessly damaged by pieces of metal travelling at a high velocity. Such fragments are considered sterile and hence the question of infection is not an important one.

The initial trials were made by Mr. H. Campbell Orr with the co-operation of Messrs. C. W. Dixey and Son, Ltd. The first method used was to pin the excised cornea to a suitably shaped mould, consisting of an acrylic ball conforming to the curvature of the eye, with holes drilled in it to accommodate the pins used to fasten the cornea. This method was discarded as the donor cornea was not held firmly enough by the pins. More elaborate devices were being considered when matters were precipitated by a patient who needed a lamellar corneal graft as an emergency measure, the only donor material available being a portion of a cornea from an eye to be eviscerated after a recent injury. The device described below gave a satisfactory answer to an urgent problem. It has the virtues of simplicity and ease of manufacture and proved so convenient that we have continued to use it with only a few minor modifications.

\section{Construction}

The appliance comprises a closely fitting cup-shaped attachment which slides easily over the top of a Tudor Thomas stand. The excised donor cornea is held between a resilient plastic ball which rests in the hollow on top of the stand and the attachment itself (Fig. 1, opposite). The attachment is pressed down till the cornea is held firmly and a set screw on the side fixes it in place (Fig. 2, opposite). 


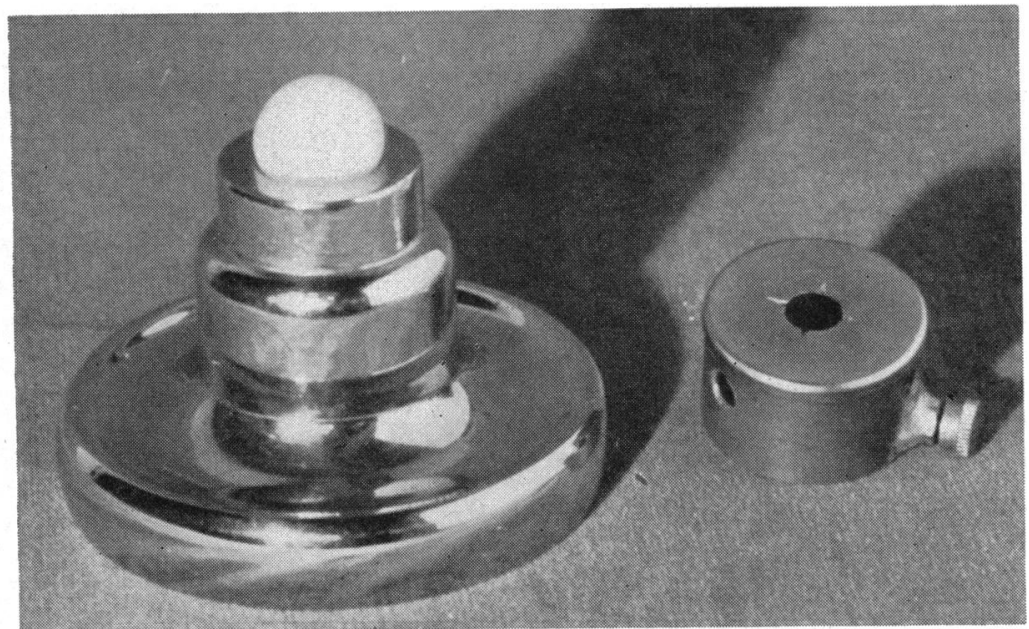

Fig. 1.-Attachment and plastic ball used for fixing excised cornea.

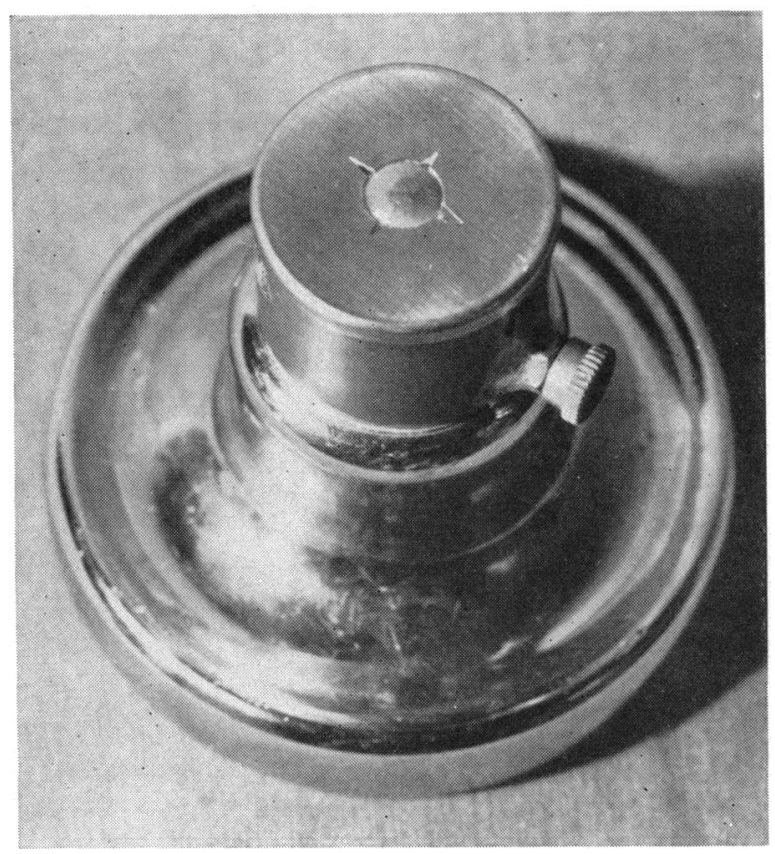

FIG. 2.-Intact enucleated eye fixed on the stand.

The circular aperture at the top is $11 \mathrm{~mm}$. in diameter and fits over the donor cornea holding it near its periphery. Four pairs of teeth cut in the edge of the aperture help to fix the cornea and prevent it from rotating while a graft is being trephined. A drop of saline between the cornea and the plastic ball provides a cushion which one can trephine against.

The teeth divide the aperture into quadrants and serve as markers for four pre-placed corneal sutures if such a technique is desired in lamellar grafting. The smooth contour of the attachment saves the sutures fouling the fingers or the gauze swabs which are normally used to hold the donor eye. The flat surface of the attachment provides a firm support for the fingers during delicate manipulations. 


\section{Methods of Use}

The device has been found so convenient that it has also been used to fix intact enucleated eyes while cutting lamellar and full-thickness grafts, in preference to the usual suture in the optic nerve technique. The globe is merely dropped into the hollow in the top of a Tudor Thomas stand and the attachment is fixed over it as described earlier, the whole procedure taking only a few seconds.

The level of the intra-ocular pressure is of importance during the cutting of a graft. The surgeon or his assistant can delicately adjust this pressure by indenting the globe with the point of a pair of forceps introduced through a hole in the side of the attachment. Three such holes are made so that one of them will always be in a convenient position whichever way the stand is rotated. The cornea can also be centred in relation to the aperture in the top of the attachment by gripping a muscle insertion on the globe through one of these holes.

We have used an attachment with a $11 \mathrm{~mm}$. aperture at this hospital for several months and find it entirely suitable for the type of work described. Sections of copper pipe were used for the barrel of the attachment, the top being made of stainless steel soldered on to the copper barrel. The whole job was done in the hospital workshop by the tinsmith, Mr. White, and could not have cost more than a few shillings. 